\title{
Facilitando la separación psicológica de las mujeres en proceso de terminar una relación de pareja violenta
}

\section{Facilitating psychological separation for women leaving a relationship with a violent partner}

\author{
Esteban Leonardo Laso Ortiz ${ }^{(1)}$, Lidia Karina Macias-Esparza ${ }^{(1)}$, \\ Roser Cirici Amell ${ }^{(2)}$ y Guillem Feixas I Viaplana ${ }^{(3)}$
}

(1) Universidad de Guadalajara, México

(2) Servicio de Salud Mental en Barcelona, España

(3) Universidad de Barcelona, España

\begin{abstract}
Resumen: La violencia de pareja contra las mujeres constituye un problema social y de salud pública. Por ello, la Terminación de una Relación de pareja Violenta (TRV) ha sido objeto de múltiples investigaciones e intervenciones en el área de la salud y los servicios sociales. Aunque hay consenso en considerar la separación psicológica (SP) como un componente crucial para la TRV, no existe una definición que permita a las y los profesionales comprender el fenómeno y orientar su intervención. En este artículo realizamos una revisión del término del proceso de SP, proponemos una definición procesual de la misma caracterizada por varias etapas clave y cerramos con recomendaciones generales para la atención que se puede brindar a las mujeres víctimas de violencia de pareja.

Palabras clave:_Violencia contra la mujer, violencia basada en el género, violencia de pareja, terminación de la relación, separación psicológica, preguntas clave.
\end{abstract}

\begin{abstract}
Partner violence against women is a social and public health problem. Hence, termination of a violent partner relationship (VRT) is the subject of multiple investigations and the goal of several interventions in the area of health and social services. In spite of the consensus in considering psychological separation (PS) as a crucial component for VRT, there is no definition that allows professionals to understand the phenomenon and guide its intervention. In this article we conducted a review of the ending of the PS process, we propose a procedural definition characterised by several key stages and close with general recommendations for the type of care that could be offered to women who are victims of partner violence.

Keywords: Gender-based violence, intimate partner violence, leaving a violent intimate relationship, psychological separation, key questions.
\end{abstract}

La correspondencia sobre este artículo deberá ser enviada a Esteban Leonardo Laso Ortiz. E-mail: estebanlaso@gmail.com

(cc) BY-NC-ND Este es un artículo Open Access bajo la licencia CC BY-NC-ND. 
Violencia de pareja contra las mujeres, violencia de género y sus implicaciones en la salud mental

El término violencia contra las mujeres alude a las formas de violencia cuyas víctimas son mujeres. Se clasifica en distintas modalidades (familiar, laboral, docente, institucional, comunitaria y feminicida) y tipos (psicológica, física, patrimonial, económica, sexual). Este tipo de violencia ha sido reconocida por la Organización Mundial de la Salud (OMS, 1996) como un grave problema de salud pública asociado con riesgos para la salud reproductiva, enfermedades crónicas, consecuencias psicológicas, lesiones y muerte (Oram et al., 2016; Trevillion et al., 2016; Velzeboer et al., 2003; World Health Organization, 2013). De todas las formas de violencia contra las mujeres, la más común es la que proviene de la pareja, mucho más que las agresiones o violaciones perpetradas por extraños o simples conocidos (OMS, 2005; UNODC Oficina de las Naciones Unidas contra la Droga y el Delito, 2013; Velzeboer et al., 2003). Así, las mujeres entre 15 y 44 años de edad corren mayor riesgo de ser violadas o maltratadas en su casa que de sufrir cáncer, accidentes de vehículos o malaria, según estadísticas del Banco Mundial citado por la Organización de las Naciones Unidas (ONU, 2008).

Asimismo, la violencia contra las mujeres por parte de sus parejas se encuentra en casi todas partes del mundo. La OMS (2005) refiere que según estudios realizados en 35 países, entre el 10\% y el 52\% de las mujeres reportaron haber sufrido maltrato físico por parte de su pareja en algún momento de su vida, y entre el $10 \%$ y el $30 \%$ haber sido víctima de violencia sexual. Dada su prevalencia, es casi inevitable que las y los profesionales de la salud mental atiendan a mujeres que viven este tipo de situación, lo que hace imprescindible que cuenten con la formación necesaria para identificarla cuando se presenta detrás de diversos trastornos o motivos de consulta (Macias-Esparza y Laso, 2017), detectar y reducir del riesgo y eventualmente contribuir a la desaparición de la violencia a través del trabajo clínico.

Por su parte, el concepto violencia de género o violencia basada en el género (gender based violence) hace referencia a la violencia que se entiende como producto de las relaciones de género, destacando la importancia del sistema sexo/género como vertebrador de la misma. En sentido estricto, este término comprende cualquier tipo de violencia ejercida por razones de género y no necesariamente aquella que tenga a la mujer como víctima; así, pudieran incluirse las acciones violentas de un hombre hacia otro o los casos en que un hombre homosexual o transgénero es víctima de violencia (transfobia u homofobia). Sin embargo, aunque se considera violencia de género a toda aquella ejercida contra una persona sobre la base de su sexo/género, dirigida tanto a hombres como a mujeres, en la práctica la inmensa mayoría de víctimas de violencia de género son mujeres, por lo cual normalmente se asocia este tipo de violencia a la dirigida contra la mujer. En consecuencia, es común utilizar como sinónimos los términos de violencia de género, violencia contra la mujer y violencia machista, lo que da a entender que son las relaciones de poder, la construcción social y el sistema sexo/género lo que la articula.

En el ámbito de la salud mental, la violencia de género tiene diversas secuelas entre las que se encuentran el trastorno por estrés postraumático, la depresión y la ansiedad, los cuales se presentan entre el $50 \%$ y $80 \%$ de las víctimas, (Blanco et al., 2004; Amor et al., 2002; Humphreys et al., 2001; Oram et al., 2016; Trevillion et al., 2016; Organización Mundial de la Salud, 2001; Pico-Alfonso et al., 2006). Se ha reconocido que sin importar cuán severos sean los daños físicos de la violencia, las consecuencias para la salud mental pueden ser tan graves, o incluso más, que para la salud física, sobre todo porque sus efectos son más prolongados (Henning y Klesges, 2003; Sagot y Carcedo, 2000). Así, según Krug et al. (2002), las consecuencias de la violencia pueden persistir aún cuando el maltrato ha desaparecido ya que mientras más severo es este, mayor el impacto en la salud física y mental de las víctimas; finalmente, el impacto en el tiempo de diferentes tipos de maltrato y de múltiples episodios de violencia parece ser acumulativo. Como consecuencia de lo anterior, es necesario que las y los profesionales de la salud estén familiarizados con los conceptos básicos del género para atender adecuadamente los casos de violencia de pareja y de género.

Sin embargo, existe evidencia de que esta necesidad no se cumple: las y los profesionales de la salud y de los servicios sociales en general no están suficientemente preparados para brindar la atención adecuada en casos de violencia de género, lo cual puede tener efectos iatrogénicos (Cirici et al., 2010). Así, es común que las mujeres busquen atención precisamente tras un momento crítico de violencia y que, por la falta de formación, la o el profesional repare únicamente en este incidente como un evento aislado, pasando por alto la historia de 
múltiples violencias, dominación, intermitencia de violencia, vínculos afectivos, etc., que ha vivido la mujer. Como resultado, el o la profesional puede aconsejar a la mujer que se separe o proceda a la terminación de la relación violenta (en adelante, TRV), creyendo que es la manera más expedita y obvia de evitar nuevos maltratos y sorprendiéndose cuando la mujer retrocede ante esa sugerencia. Considerando el episodio violento como evento único, es lógico que las y los profesionales no comprendan las decisiones de las mujeres y las estigmaticen:

Por ello suelen aparecer las frases "si a mí me pasara..." "yo no aguantaría que..." porque pensamos que a la mujer lo que le ha ocurrido es que en alguna ocasión su pareja la ha insultado, humillado o golpeado y no entendemos como no hace algo contra ello. Invisibilizamos todo el proceso anterior, que es la auténtica construcción de la violencia (lo que vemos serían las consecuencias), no viendo el proceso de victimización que lleva a una persona a convertirse en víctima. (Paz, 2011, p. 449).

Por tanto, la falta de conocimiento sobre las relaciones de género y su influencia en la violencia de pareja conduce a las y los profesionales a expectativas poco realistas sobre el resultado de su intervención y de las razones por las cuales las mujeres permanecen en relaciones violentas (Nicolaidis et al., 2005). Es común que las y los profesionales estigmaticen, culpen y responsabilicen a las víctimas por la violencia recibida y por permanecer en las relaciones (Crowe y Murray, 2015; Morse et al., 2012), todo lo contrario de lo que necesitan en momentos de vulnerabilidad: que no las juzguen, que sean compasivos y sensibles, que muestren una comprensión de la complejidad de la violencia y comprendan su naturaleza a largo plazo, sus características sociales y psicológicas y la dificultad de una resolución rápida, así como que eviten medicalizar el problema. Todo ello conduce a que las mujeres rehúyan acudir a los servicios de salud por temor a recibir respuestas inadecuadas por parte de quienes las atiendan (Feder et al., 2006; Robinson y Spilsbury, 2008), lo que las vulnerabiliza aún más.

\section{“Si sufre violencia, sepárese”: por qué recomendar la separación puede ser iatrogénico}

Es habitual que, tanto en la práctica clínica como en los servicios sanitarios y sociales, se considere que las mujeres deben eliminar rápidamente la violencia de sus vidas por lo que las intervenciones se orientan casi siempre hacia la forma más expedita de conseguirlo: la terminación de la relación violenta o TRV (McCloskey et al., 2006; Morse et al., 2012; Rees et al., 2014). Así, Morse et al., (2012) señalan que el 71\% de las participantes de su estudio informaron que los profesionales sanitarios les insinuaron que debían terminar la relación violenta, mientras que el $37.5 \%$ del total afirmó que el profesional les aconsejó expresamente dejar a la pareja violenta.

Sin embargo, es común que las mujeres oscilen entre separarse y regresar con su pareja o que, tras proponerse la separación en un primer momento terminen arrepintiéndose, conductas que son entendidas generalmente como "dificultades personales" suyas. Al ignorar el trasfondo de desigualdad y violencia estructural sobre el que emerge la violencia de pareja contra la mujer y la desventaja en que ésta se encuentra (porque, por ejemplo, carece de los recursos económicos necesarios o tiene que hacerse cargo de sus hijos sin apoyo del padre "porque esa es la responsabilidad de la mamá"), esta conceptualización "psicologiza" una problemática sociocultural, responsabilizando exclusivamente a la víctima y ofreciéndole una alternativa en muchas ocasiones impracticable o impensable desde su confusión y ambivalencia emocional; una alternativa, la de separarse o terminar la relación, que se entiende como un evento único, aislado, voluntario y decisivo.

Este consejo de "sentido común", se apoya en tres supuestos erróneos:

1. Que la decisión de terminar la relación conlleva una separación más o menos inmediata,

2. Que dicha decisión depende de la voluntad de la mujer, (sin considerar aspectos sociales y estructurales) y

3. Que se concreta en una acción única, inexorable y definitiva (como si las mujeres víctimas de violencia de género en la pareja pudieran simplemente decir "He decidido terminar la relación mañana a las 5 de la tarde en punto"). 
Y son erróneos porque, como exponemos a continuación, la terminación de la relación violenta debe concebirse como un proceso en el cual intervienen diversos factores psicológicos y sociales, ante todo los de género. En concreto, para que la mujer pueda decidirse a terminar la relación violenta y, sobre todo, persistir en esta decisión, debe necesariamente pasar por una etapa previa: la separación psicológica, concepto que brilla por su ausencia en la concepción de "sentido común" que parecen abrigar la mayoría de interventores.

\section{Por qué la terminación de la relación violenta es un proceso: dificultades de abandonar la relación derivadas de las diferencias de género}

Si bien los primeros estudios datan de la década de los 70, el tema de la terminación de una relación violenta ha experimentado un auge en la última década, investigándose bajo términos como "abandono de la relación violenta", "escape de la relación violenta", "abandono de la pareja violenta", "finalización de la violencia" y sus equivalentes en habla inglesa. Es sólo a mediados de los 80 que comienza a entenderse como un proceso más que como un acto aislado al ponerse en evidencia dos hechos:

1) las situaciones de violencia de género en las parejas son crónicas y de larga evolución: según datos de Krug et al. (2002) y Navarro (2015) la duración promedio de estas relaciones es de seis años (aunque las mujeres jóvenes tienden a terminarla antes) pero puede extenderse hasta los 13 años, según el estudio de Amor et al. (2002);

2) la terminación de la relación violenta conlleva múltiples intentos y separaciones temporales: en promedio ocurren de 5 a 7 separaciones transitorias antes de la terminación definitiva (Anderson y Saunders, 2003; Burman, 2003; Griffing et al., 2002; Navarro, 2015; Landenburger, 1989; Wuest y Merritt-Gray, 1999).

De lo anterior se sigue que por violenta que sea la relación, abandonarla conlleva grandes dificultades para la mujer, que se visibilizan al adoptar una perspectiva procesual y de género que resalta la estructura patriarcal de las sociedades contemporáneas. La complejidad del fenómeno precisa conocer que existen algunos factores sociales y económicos que toman un papel importante en la aparición de la violencia de pareja y su terminación; como son el acceso de las mujeres a la educación; las normas y mandatos culturales sobre el género, así como el apego o compromiso con la pareja (Dalal, 2011; Vyas y Watts 2009). En lo económico, destacan los factores de la brecha salarial por género, el suelo pegajoso (sticky floor) y el techo de cristal (glass ceiling) (OCDE, 2014; ONU MUJERES, 2013; World Economic Forum, 2020). La brecha salarial es la diferencia de sueldos entre hombres y mujeres al realizar un mismo trabajo. El suelo pegajoso se refiere a la dificultad que experimentan las mujeres para incorporarse al mundo laboral debido a que se les responsabiliza del trabajo maternal y doméstico no remunerado, lo cual influye decisivamente para que abandonen sus trabajos o busquen jornadas reducidas y de menor paga, debido a la doble jornada y a la escasa o nula corresponsabilidad de sus parejas y de las insuficientes medidas de conciliación de la vida laboral y familiar. El techo de cristal se refiere al acceso limitado a los puestos de poder que impide a las mujeres su crecimiento profesional para obtener un ascenso laboral al interior de las organizaciones profesionales, sociales o económicas.

En lo cultural, como señalan Ferrer y Bosch (2013), existe una socialización diferenciada que contempla mandatos de género distintos para hombres y mujeres y, en el caso de estas últimas, posiciona al amor como eje vertebrador del proyecto de vida y del "ser para otros" exaltando su capacidad de entrega, su papel como cuidadora y responsable del bienestar de otros/as sin esperar nada a cambio e incluso renunciando a las propias necesidades o deseos (o, en otras palabras, privilegiando la comunión por sobre la agencia; Laso 2017). Entendido como mandato de género que remite al altruismo y a la incondicionalidad, dejando de lado la reciprocidad, este "amor" explica el alto nivel de compromiso con la relación que suelen tener las mujeres que mantienen una relación de pareja en la que existe violencia; así, Anderson y Saunders (2003); Rhatigan et al. (2006) mencionan que el nivel de compromiso con la relación y los sentimientos de amor dificultan el proceso de TRV.

Además, como señala Navarro (2015), durante el primer año de ruptura, las víctimas de violencia de género en la pareja se ven obligadas a resolver varias tareas, de por sí complicadas y estresantes, al tiempo que 
lidian con dificultades añadidas como la violencia post-separación o las pérdidas económicas (reducción de sus ingresos y pérdida de la vivienda). Además, generalmente son las mujeres quienes deben asumir las responsabilidades económicas y de crianza de los hijos e hijas reconfigurándose como familia monoparental, a la vez que deben procurar que éstos comprendan y asimilen los cambios vividos (de residencia, de amigos, de colegio, de vecinos, etc.) en un momento en que la mujer misma puede estar seriamente afectada.

Por todos estos obstáculos, terminar la relación violenta no es prácticamente nunca un evento voluntario, único e irreversible; y por tanto, tampoco es necesariamente la mejor o más rápida opción para terminar la violencia de la pareja. De hecho, y según se ha demostrado, la separación (tanto física como psicológica) es un importante factor de riesgo para la incidencia de violencia letal (Campbell et al., 2003; DeKeseredy et al., 2007; DeKeseredy et al., 2004; Dobash et al., 2007), por lo que en muchos casos permanecer con la pareja puede ser a corto plazo la alternativa menos peligrosa para las mujeres.

En definitiva, entender de forma simplista la terminación de la relación violenta como un acto de voluntad favorece la culpabilización y patologización de las víctimas, pues conduce a pensar que "la mujer no quiere cambiar", "le gusta el maltrato y/o es masoquista", "obtiene ganancias secundarias de la situación", "es pasiva y no tiene fuerza de voluntad, ni autoestima", "es tonta", etc. Por el contrario, pensar la terminación de la relación violenta en términos de proceso permite considerar a las víctimas como sujetos activos en una sucesión compleja y no necesariamente lineal de toma de decisiones que el interventor puede fomentar y facilitar independientemente del contexto en que tome contacto con la mujer victimizada.

\section{La separación psicológica: precondición de la terminación de la relación violenta}

Como hemos apuntado, para que la terminación de la relación violenta sea sostenible ha de atravesar un hito, la separación psicológica, que es el tema de este artículo y que, pensamos, debe convertirse en el centro de gravedad de las intervenciones para asistir a las mujeres víctimas de violencia desde una perspectiva sensible al género.

En primer lugar, es claro que las distintas formas de separación -ya sea legal, física o psicológica- son clave dentro de la terminación de la relación; pero la sola presencia de algún tipo de separación no hace inminente la terminación de la relación, ya que una pareja puede estar separada físicamente, como en el caso de relaciones a distancia, y aún así seguir fuertemente comprometidos en continuar el vínculo. Por ende, entendemos por separación física el acto, temporal o definitivo, por el cual la mujer deja de cohabitar o de tener contacto físico con la pareja. Las separaciones físicas temporales son frecuentes y el regreso a la relación violenta no debe entenderse como signo de debilidad, sino como un ensayo, de ser el caso, para la ruptura final. La evidencia sugiere que el factor determinante para la ruptura definitiva de la relación es la existencia de una separación psicológica, concepto que se ha planteado en la literatura como un elemento crucial para la TRV (Anderson y Saunders, 2003; Khaw y Hardesty, 2009; Peled et al., 2000; Walker et al., 2004); para la separación física definitiva en casos de violencia sexual por parte de la pareja (Block y DeKeseredy, 2007; DeKeseredy y Joseph, 2006; DeKeseredy et al., 2004) y la posterior recuperación de la mujer (Roca-Cortés et al., 2005; Roca-Cortés et al., 2007).

Uno de los modelos más elaborados que mencionan la separación psicológica es el propuesto por Roca-Cortés et al. (2007) con respecto al proceso de recuperación que viven las mujeres tras una relación violenta. Estudios recientes aportan una definición conceptual de la separación psicológica como aquella en la cual "la mujer rompe los vínculos de amor y deseo hacia la relación de pareja y todo lo que representa esta relación en su proyecto vital y en su identidad social" (Roca-Cortés et al., 2015, p. 56).

Scheffer y Renck, (2008) en relación al proceso de TRV también señalan la existencia del abandono o separación psicológica, apuntando la posibilidad de que dicho proceso no se concluya hasta después de la ruptura física. DeKeseredy et al., (2004) describen que en el intento de salir de una relación violenta las mujeres se separan emocionalmente, realizando acciones paralelas como dejar de cohabitar con la pareja, tramitar y mantener una denuncia e iniciar y/o finalizar una separación legal o divorcio. Desde el contexto de la violencia sexual, definen la separación emocional como la negación de la mujer a mantener relaciones sexuales u 
otro tipo de intercambios íntimos con su pareja. De acuerdo con estos autores, la separación emocional es el principal predictor en la terminación de una relación sexualmente violenta.

Por su parte, Khaw y Hardesty (2009) resaltan la necesidad de distinguir entre la presencia física y la presencia psicológica o simbólica de los miembros participantes en una relación, refiriendo que la ausencia psicológica -equivalente a la separación psicológica- se halla presente en el proceso de TRV. También postulan la existencia de dos tipos de límites llamados "ambiguos", entendidos como un estado de dificultad para definir el rol de la pareja o ex pareja durante el proceso de separación, destacando que la ambigüedad de límites no ha sido estudiada como un mecanismo específico en el proceso de terminación de la relación con una pareja abusiva y que dicha ambigüedad puede ser un factor que afecte la capacidad de las mujeres para permanecer separadas de sus parejas. Postulan la existencia de dos tipos de límites llamados ambiguos:

En el límite ambiguo Tipo I, la persona está ausente físicamente pero mantiene la presencia psicológica ya que se siente unida a su pareja mientras no tenga lugar la ruptura definitiva. La trayectoria de terminación comienza con la separación física y prosigue con la psicológica.

En el límite ambiguo Tipo II la mujer está físicamente presente pero emocionalmente ausente. La trayectoria de terminación se inicia por la separación emocional y concluye con la separación física.

Más tarde, estas mismas autoras (Khaw y Hardesty; 2014) sugieren que la separación psicológica es previa a la etapa de acción del modelo transteórico, refiriendo que las mujeres pueden ser más capaces de desconectar psicológicamente de sus parejas maltratadoras, facilitando el proceso de despedida emocional, para pasar a la fase de acción.

Por otro lado, Anderson y Saunders (2003) utilizan el término abandono emocional y mencionan que la terminación de una relación empieza con cambios emocionales y cognitivos antes de que tenga lugar la separación física. De acuerdo a estos autores, la terminación de una relación violenta es la continuación de un proceso que comienza en un nivel emocional y cognitivo, que transcurre mientras la mujer continúa en la relación y se extiende mucho más allá de la separación física.

Como se puede apreciar, estos aportes han ido convergiendo independientemente en el concepto de separación psicológica pero han permanecido inconexos y fragmentarios. De ahí la propuesta de este texto: integrarlos en una definición operativa que contribuya a precisar los elementos y fases del proceso de separación de una relación violenta y el papel que en éste desempeña la separación psicológica.

\section{Conceptualizando la separación psicológica.}

La propuesta de definición de la SP presentada se basa en un estudio empírico previo realizado por Macias-Esparza, $(2017)^{1}$. Elegimos el término separación psicológica por considerarlo más amplio que separación emocional, ya que lo psicológico incluye emociones, acciones o comportamientos (por ejemplo, irse de casa), cogniciones y planes o proyecto vital.

Proponemos que el proceso de separación psicológica se encuentra anidado en otros dos procesos de mayor alcance: el de instauración y mantenimiento de la violencia y el de terminación de la relación. El considerar estos tres procesos como distintos pero relacionados entre sí, permite visualizar que la toma de decisiones de un proceso es dependiente de las decisiones tomadas con anterioridad como parte de otro; y, además, que estas decisiones dependen de la respuesta que la mujer brinda a ciertas preguntas clave que caracterizan cada etapa y que explicaremos más adelante.

En concreto, la separación psicológica ocurre dentro de otro proceso que lo abarca, el de TRV, el cual a su vez ocurre dentro del proceso primario de aparición y evolución de la violencia de género en la pareja, lo cual es lógico si se observa que para que pueda darse una separación ha de ser motivada por la existencia de violencia de pareja, y para que pueda darse ésta, ha de existir previamente una relación de pareja. Cabe mencionar que

1 Este documento comunica parte de los resultados de los estudios que componen la tesis doctoral Dilemas implicativos y separación psicológica de mujeres en proceso de terminación de una relación violenta, Macias-Esparza, 2017. 
varios autores han sugerido que la separación psicológica es un proceso dentro de otro (Anderson y Saunders, 2003; Enander, 2011; Enander y Holmberg, 2008; Khaw y Hardesty, 2007, 2009, 2014; Scheffer y Renck, 2008), si bien no de forma explícita como en la presente propuesta.

A partir de lo anterior, definimos la separación psicológica como:

a) Un proceso anidado dentro del proceso de TRV, a su vez inmerso en el proceso de instauración de la violencia de género en la pareja,

b) Que se conforma de un conjunto de estrategias y cambios cognitivos, conductuales y emocionales (que sirven como indicadores) que vive la mujer a partir de que se inicia la violencia dentro de su relación de pareja,

c) Cambios o estrategias que se agrupan en dos subfases:

a) Subfase de disminución o minimización del malestar o daño;

b) Subfase de separación psicológica propiamente dicha.

d) Dichos cambios o estrategias incrementan la distancia relacional con su pareja maltratante (ya sea de forma intencionada o no, dependiendo de la subfase en que se desarrollen);

e) Pueden desembocar, inicialmente, en el deseo de poner fin a las consecuencias de la violencia y, eventualmente, en el deseo y/o la decisión por parte de ella de poner fin a la relación;

f) A partir de tomar dicha decisión la mujer planifica y/o realiza cambios para llevar a cabo bien la terminación de la relación, bien (en el caso de que sea posible) la terminación de la violencia y los síntomas asociados a esta sin abandonar la relación.

\section{Las fases de la terminación de la relación violenta}

Proponemos que, para ser capaz de terminar la relación violenta, la mujer debe atravesar la secuencia que se plasma en la figura 1 y se explica a continuación:

1. Establecimiento de la relación de pareja;

2. Emerge la violencia como forma de relación (es decir, la mujer sufre repetidos episodios de violencia física, psicológica o sexual por parte de su pareja);

\section{Inicia el proceso de instauración de la violencia}

3. Eventualmente, la mujer identifica la violencia o le da nombre, respondiendo a preguntas clave como: ¿Esto es violencia? ¿En mi relación de pareja está presente la violencia?”

4. Evalúa si existe un alto nivel de apego o compromiso en la relación y si la violencia es coherente con su identidad y sistema de creencias y valores, mediante preguntas clave como: “¿Quiero/debo seguir con la relación aun cuando sea violenta? ¿Vale la pena continuar con esta relación?”

5. Si existe un alto nivel de apego o compromiso, la mujer mantiene la esperanza de que su pareja pueda cambiar o de que la relación pueda mejorar; la pregunta clave es “¿Puede mi pareja dejar de ser violento o puede cambiar la relación?"

\section{Inicia el proceso de separación psicológica (subfase 1: minimización del malestar o daño).}

6. La mujer intenta cambiar a la pareja a través de dos estrategias:

a) si es una relación simétrica, mediante la coerción, la denuncia, las amenazas de TRV o incluso mediante el ejercicio de la violencia bidireccional; o bien, 
b) si es una relación complementaria, a través de acciones como la sumisión y la complacencia, o la búsqueda de soluciones alternativas como la solicitud de terapia de pareja, asesoría espiritual, etc.

7. La pregunta clave de esta fase es “¿haciendo esto, desaparecerá la violencia?” Por tanto, la mujer evalúa si la estrategia utilizada ha tenido éxito, considerando las siguientes posibilidades. La violencia:

a) desaparece,

b) se reduce a un nivel que la mujer considera tolerable,

c) continúa igual,

d) empeora.

8. Si no tienen éxito estas estrategias, es decir, si la violencia continúa igual o empeora, la mujer puede empezar a cuestionarse si no hay posibilidad de cambio; las preguntas claves son: “¿la relación puede cambiar?, ¿mi pareja puede cambiar? ¿Quién debe hacer algo para que la violencia desaparezca?” y continúa sometiendo dichas cuestiones a experimentos que le permitan darse una respuesta.

9. Identifica las consecuencias de la violencia a la luz de la imposibilidad de cambio. La pregunta clave es "¿qué consecuencias ha tenido la violencia en mi vida y en la vida de los seres que quiero?"

\section{Continúa el proceso de separación psicológica (subfase 2: separación propiamente dicha).}

10.Eventualmente, deja de creer en la posibilidad de que su pareja cambie o la relación mejore (es decir, responde negativamente a dicha pregunta clave).

11.Decide terminar la relación (puede ser mediante la planificación de TRV o mediante una huida repentina).

\section{Inicia el proceso de terminación de la relación violenta}

Como se puede apreciar, casi cada una de las etapas incluye una pregunta clave como "esto que estoy viviendo, ¿es violencia?” o “¿puede mi pareja dejar de ser violenta?” Esta pregunta clave puede entenderse como la hipótesis tácita que guía el comportamiento de la mujer a lo largo de dicha etapa, permitiéndole integrar la información sobre los actos de su pareja, sus motivaciones y/o implicaciones, para adoptar eventualmente una postura al respecto y tomar una decisión. Así, entre el sinnúmero de cosas que la mujer debate consigo misma en cada etapa, va diferenciándose una duda que sintetiza el tema fundamental de la misma y que se convierte en el prisma con que contemplará los hechos en lo sucesivo.

\section{La separación como conjunto de procesos anidados y el modelo transteórico del cambio}

El antecedente más próximo a esta propuesta de la separación como resultado de varias etapas anidadas, cada una de las cuales se caracteriza por una pregunta clave, es la secuencia que adelantan Burkitt y Larkin (2009) con base al modelo transteórico del cambio. Los autores analizan la forma en que las cinco etapas de dicho modelo (precontemplación, contemplación, preparación, acción y mantenimiento) se manifiestan en la experiencia de las mujeres víctimas de violencia de género por parte de su pareja e identifican que el desafío de la tercera, preparación, consiste en abandonar la esperanza de que su pareja cambie, mientras que el de la quinta, mantenimiento, equivale a "lograr la autonomía y la separación del yo" (Burkitt y Larkin, 2009, p. 282). Existe una coincidencia entre estas etapas y las fases que integran la presente propuesta; en concreto, la etapa de preparación equivale a la pregunta clave de la subfase de minimización del daño o malestar: "¿puede 
Figura 1. Diagrama flujo de violencia en relación pareja

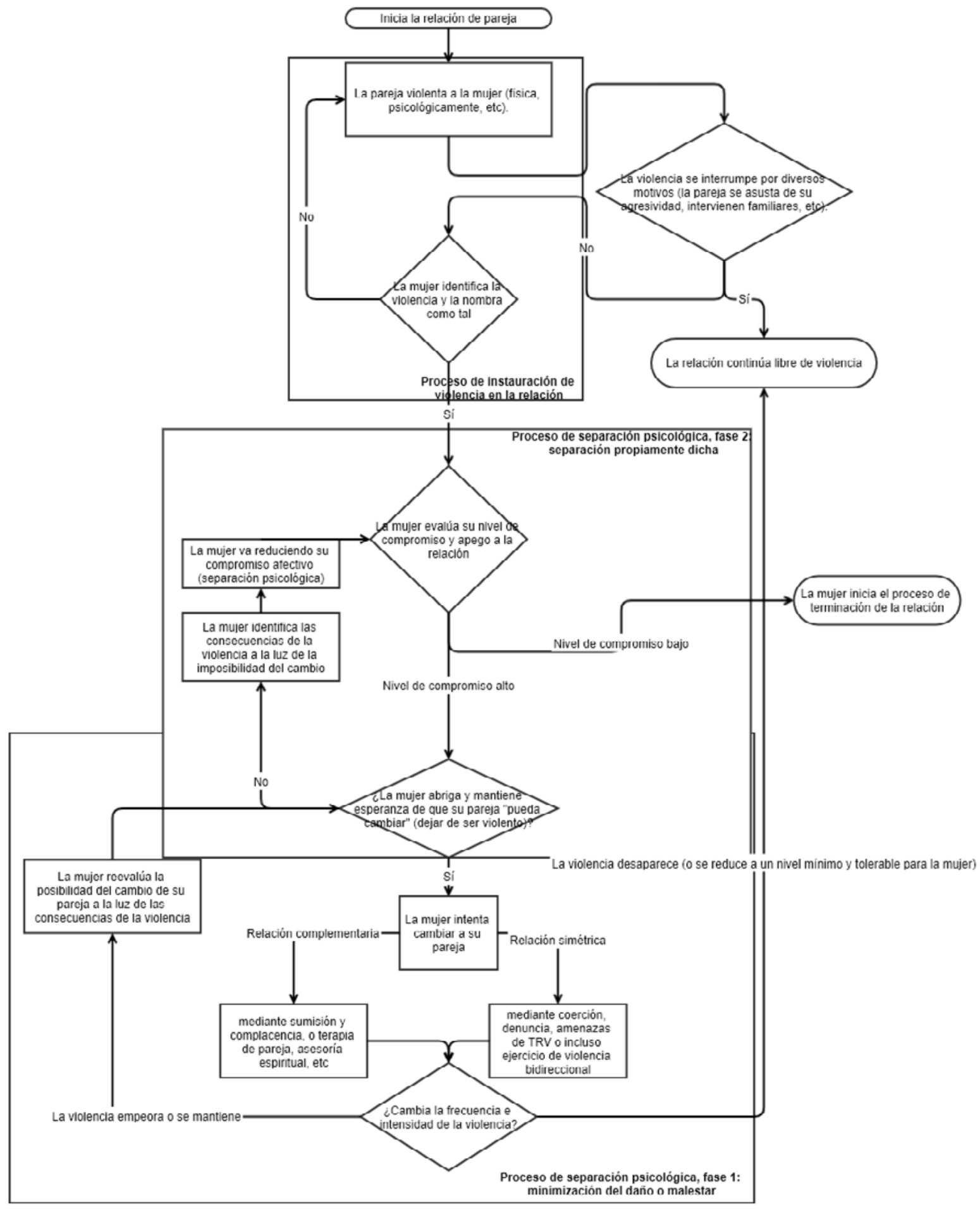

cambiar mi pareja?", y la de mantenimiento, a la fase final de la terminación de la relación una vez alcanzada la separación psicológica.

Esta coincidencia ofrece cierta confirmación para la presente propuesta; la cual, sin embargo, va más allá de la mera aplicación del modelo transteórico del cambio al problema de la violencia de género en pareja ya que, 
por una parte, incorpora explícitamente los desafíos que la mujer ha de superar a cada paso de su proceso de terminación o cambio de la relación; y, por otra, reconoce el carácter anidado de las fases que lo integran, lo que permite entender los frecuentes retrocesos de las mujeres y el prolongado período que suelen necesitar para romper con las relaciones de violencia.

\section{Claves mínimas para la atención a mujeres que viven violencia de género en la pareja}

Además de sugerir líneas para la investigación, de esta propuesta de la terminación de la relación violenta como conjunto de procesos anidados, el más importante de los cuales es la separación psicológica, se siguen algunas recomendaciones para la intervención con mujeres víctimas de violencia de género por parte de su pareja, que pasamos a reseñar.

\section{Prevención: educar a la red de apoyo y no sólo a la víctima}

En primer término, el comprender que la violencia tiene que repetirse para quedar instaurada como forma de relación sugiere que cualquier intervención que la interrumpa in statu nascendi puede servir para prevenirla, conduciendo bien a la continuación de la relación sin violencia, bien a la ruptura de ésta antes de que el nivel de compromiso emocional e instrumental de la mujer lo haya vuelto prohibitivo. Haciendo un paralelismo con una estrategia que se ha demostrado eficaz para prevenir el bullying (Midgett et al., 2015), puede ser provechoso enseñar a discernir la violencia de género en pareja no sólo a las potenciales víctimas sino sobre todo a sus familiares y amigos cercanos (por ejemplo, aprovechando esquemas como la "escuela para padres" para educarlos sobre la violencia en el noviazgo), de modo que puedan intervenir para concienciar a la mujer y/o su pareja, cortándola de raíz. Habida cuenta de que la violencia de género suele ser un despliegue "homosocial", esto es, destinado a que los demás varones reconfirmen la masculinidad de quien la ejerce (Kimmel, 2013), estos programas psicoeducativos podrían ser más eficaces si se destinan específicamente a ellos.

Una vez instaurada la violencia como patrón relacional, el siguiente punto susceptible de intervención es cuando la mujer reconoce que está siendo victimizada; es aquí donde, desde nuestro punto de vista, se ha dirigido la mayoría de las campañas de concienciación. Consideramos indispensable facilitar el empoderamiento de las mujeres y su capacidad de detectar la violencia que sufren. Sin embargo, al tomar únicamente este modo y no el anterior como el núcleo de las políticas sociales, se podría dificultar inadvertidamente la reducción de la violencia porque, llegado a este punto, el nivel de compromiso y/o dependencia (económica, social, etc.) de la mujer puede ser mayor que su capacidad de ejercer su derecho a ser respetada y no violentada. Por ende, una primera recomendación es destinar recursos educativos no sólo a las mujeres sino también a las personas que son testigos o espectadores de la violencia (bystanders) para que puedan intervenir activamente en identificarla, nombrarla y obstaculizarla.

Entre estos circunstantes es crucial incluir a los y las interventoras de los sistemas de salud y educación (enfermeras, doctoras, educadoras, etc.), los cuales deben recibir formación especializada para comprender las características que distinguen la violencia de pareja de otras formas de violencia por parte de personas con las cuales no se tiene un vínculo afectivo y que son, por ende, más fáciles de romper o modificar. Algunas de estas características son: la concurrencia de distintos tipos de violencia (física, psicológica, patrimonial, sexual...) dentro de la misma relación; la manifestación cíclica y hasta cierto punto predecible de las agresiones; la presencia de dependencia emocional y económica; el progresivo aislamiento de la víctima de sus redes de apoyo; la complejidad y ambivalencia de los sentimientos hacia el agresor que complican la toma de decisiones; y el papel de las diferencias de género como posibilitadoras y articuladoras de estas violencias.

A nuestro juicio, el punto más importante en esta formación debería ser que la ruptura de una relación violenta es un proceso complejo y recursivo que requiere de una secuencia de pasos, cada uno de los cuales puede ser favorecido o entorpecido según la manera en que sea abordado. Como hemos advertido, en línea con lo sugerido por el modelo transteórico del cambio (Prochaska y DiClemente, 1982, 1994), esta concepción procesual permite interpretar las frecuentes "recaídas" (el que la mujer regrese con su pareja después de un in- 
tento de separación o que acuda a sacarlo de la reclusión retirando una denuncia que ella misma interpuso, por ejemplo) no como fracasos sino como ensayos que le permiten hacer acopio de valor, recursos y estrategias de cara a la terminación definitiva. Todo lo anterior puede ayudar al interventor a enfrentar los inevitables traspiés y obstáculos con la paciencia y empatía indispensables para ser un apoyo (tal y como ocurre con el trabajo en adicciones, Cunillera, 2006).

\section{Dos objetivos fundamentales: primum non nocere y facilitar el tránsito por cada etapa del proceso}

Esta perspectiva procesual tiene una implicación más profunda que atañe a la postura que los interventores pueden adoptar ante las mujeres víctimas de violencia que los consultan, a los objetivos específicos que pueden potenciar sus intervenciones y a la manera en que éstas deben llevarse a cabo para facilitar el cambio reduciendo el riesgo. Desde nuestro punto de vista, los y las interventoras pueden posicionarse como acompañantes de este proceso, variando su estrategia en función del punto específico en que se encuentre la mujer dentro del mismo y su táctica de acuerdo con la regularidad con que esta acuda a su consulta, pero conservando dos objetivos claros: minimizar el riesgo y propiciar la resolución de la pregunta fundamental de la mujer en el punto de decisión en que se halla.

En cuanto a estos, el primer y crucial principio que guíe la acción del interventor debe ser el de primum non nocere: preservar la seguridad de la mujer y minimizar el riesgo de que sufra nuevos episodios de violencia (Rodríguez Gimena et al., 2008). Esto incluye evitar la revictimización, por ejemplo, no culpabilizando a la mujer de la violencia y señalándole con claridad que nada la justifica, que nadie la merece ni es responsable de que otra persona se crea con el derecho de maltratarla, etc.; y se extiende a no proponerle que termine la relación violenta o se separe de su pareja sin valorar antes la probabilidad de que éste responda agrediéndola ni asegurar medidas de protección y planes de contingencia. Pero además de este objetivo negativo (en tanto que consiste en eludir un mal desenlace) el interventor debe tener un objetivo positivo, algo a lo que apuntar con sus actos que no sea necesariamente una separación o ruptura de la relación (cosa que podría, o no, traer consigo la mejora de sus condiciones o calidad de vida a corto o mediano plazo).

Es aquí donde cobra valor la propuesta de una secuencia de procesos anidados, centrados en la separación psicológica y jalonados por la pregunta clave que la mujer debate consigo misma en cada uno, ya que nos permite proponer que el objetivo positivo de los y las profesionales debe ser facilitar la toma de decisiones de la mujer con respecto a su relación violenta, cosa que se logra facilitando el tránsito de aquella por la etapa concreta en que se encuentra. Y como cada etapa está marcada por una duda crucial, la manera más directa y respetuosa de hacerlo es ayudando a la mujer a articularla de forma que pueda hacer acopio de evidencia relevante de la relación, la conducta de su pareja y su propia vivencia para responderla más allá de toda duda; esto es, en palabras de Kelly (1970), asistiéndola a diseñar un "experimento conductual”.

Por ejemplo, si la consultante se halla inmersa en la primera fase, la de instauración de la relación violenta, la interventora puede propiciar que articule la pregunta nuclear de la misma, "esto que él me hace ¿es violencia?", para diseñar colaborativamente experimentos conductuales que, sin ponerla en riesgo, les permitan obtener evidencia sobre la conducta del marido para desvanecer las dudas que mantienen a la mujer sometida a la relación. O si se halla en la segunda, que ponga en palabras su inquietud acerca de si su pareja puede cambiar y dejar de ser violento para que pueda interpretar su conducta a la luz de esta hipótesis. De esta forma, y en concordancia con los modelos constructivistas (Mahoney, 2003; Neimeyer, 2009) y con la entrevista motivacional (Miller y Rolnick, 2013), el interventor pasa de darle información o consejos desde una posición de autoridad a acompañarla a transitar lo más rápida y saludablemente posible por el camino en que ya se encuentra.

\section{Estrategia: "ser fiel a la experiencia de la relación"}

A nuestro juicio, la mejor manera de concebir este acompañamiento es como un "asistente de investigación" que la ayude a "precisar el tema; familiarizarse con él; observar cuidadosamente; formular hipótesis...; hacer pruebas preliminares; relacionar sus datos con sus predicciones... generalizar cautelosamente y revisar su 
pensamiento a la luz de la experiencia" (Kelly, 2001, p. 46). Desde esta postura, exponemos esta estrategia a las consultantes diciendo: "se trata de que puedas ser fiel a tu propia experiencia de la relación y que sea eso lo que guíe tus decisiones; yo voy a ayudarte a que escuches al máximo esa experiencia y actúes en consecuencia, manteniendo a ti y a tus hijos/hijas o allegados seguros".

Para aplicar esta estrategia, el interventor tiene presentes, en cada sesión, los dos objetivos generales: primero, asegurarse de que la mujer y sus allegados se encuentran seguros y de que sus acciones no los ponen en riesgo, y segundo, ayudarla a diligenciar la etapa del proceso en que se encuentra. A su vez, este segundo objetivo requiere:

- Que esté familiarizado con la estructura y etapas del proceso de instauración y cese de la violencia;

- Que sea capaz de solicitar o reconocer la información relevante y organizarla para identificar la etapa precisa en que la mujer se encuentra;

- Que conozca la pregunta nuclear de dicha etapa y sepa ayudar a la mujer a articularla a su ritmo, desde su propia experiencia y lenguaje (no como una imposición externa);

- Que sepa ayudarla también a diseñar situaciones que le brinden datos para irla dirimiendo sin ponerse en peligro;

- Y, finalmente, que la asista a interpretar estos datos y contrastarlos con su hipótesis para responderla definitivamente y tomar las decisiones pertinentes, avanzando en el proceso de separación o cambio de la relación violenta.

Además de estos saberes y destrezas, el interventor debe contar, por supuesto, con conocimiento básico sobre el género y sus implicaciones en la violencia de pareja, los tipos de violencia posibles, los niveles de riesgo y cómo evaluarlos, las alternativas legales y de apoyo social o salud a los que la mujer puede acudir según sus necesidades, y demás facetas específicas de esta problemática. Equipado con estos recursos, puede diseñar su plan de tratamiento de acuerdo con las posibilidades, ritmo y regularidad de la consultante, creando una táctica ad hoc para cada caso.

\section{Táctica: adecuar las intervenciones al ritmo de la consultante}

Las variables más importantes para crear esta táctica son el ritmo y la regularidad con que la consultante puede o quiere acudir con el interventor, lo cual dependerá, entre otras cosas, de la institución en la que ocurran las citas (hospital, centro de salud primaria, consulta psicológica privada...) y del rol que el interventor tenga ante la consultante (enfermera, médica, asesora legal, psicóloga...) De acuerdo con estas variables, el interventor procede a escanciar sus acciones adaptándose a las posibilidades de la mujer sobre una dimensión que va de una psicoterapia con sesiones regulares pactadas de antemano a una intervención "de sesión única" que saca el máximo provecho de un encuentro breve y ocasional (Slive y Bobele, 2013).

Para esto, los dos objetivos mencionados y las preguntas nucleares de cada etapa del proceso de separación o cambio de la relación se pueden a su vez subdividir en pasos más asequibles en una o dos sesiones que de todas formas impulsen a la mujer a avanzar. Por ejemplo, el interventor puede plantearse como meta de la sesión indagar las condiciones de vida de la mujer y crear junto con ella un plan de contingencia por si la violencia empeora, dejándola al final del encuentro con algo tangible que la motive y proteja sin importar si regresa o no a la consulta. Así, poner en palabras el sordo malestar o las vagas dudas de la mujer de modo que se aproxime a articular su pregunta básica, u organizar la información de que dispone para facilitar una más clara interpretación de la conducta del marido, son ejemplos de sub-metas valiosas que, aunque limitadas, aceleran el proceso en que la mujer se halla inmersa.

\section{Conclusión}

En este texto, y tras un análisis de la literatura relevante, hemos presentado una propuesta de definición operativa y procesual de la separación psicológica como parte del proceso de terminación o cambio de una relación violenta. Sostenemos que esta propuesta, plasmada en un diagrama de flujo de las fases del proceso y 
la pregunta nuclear que franquea el paso de una a otra, puede servir de orientación para las y los profesionales de la salud mental y los servicios sociales que brindan atención a mujeres que viven una relación de violencia de género en la pareja.

Para ello, hemos analizado las características específicas de la violencia en la pareja y las razones por las cuales es imprescindible incorporar el género como categoría de análisis y contar con una visión que considere tres procesos anidados: el de instauración de la violencia, el de terminación de la relación violenta y el de separación psicológica.

En base a esta visión procesual hemos propuesto unas recomendaciones generales para mejorar la atención que se brinda a las mujeres víctimas de violencia de pareja, los cuales atañen a:

- La importancia de circular la información preventiva entre las redes de apoyo de las mujeres y los espectadores (bystanders), sobre todo varones, de la violencia;

- Los dos objetivos que debe perseguir toda intervención: primum non nocere, es decir, primar la seguridad y facilitar el tránsito de la mujer por la etapa específica del proceso de terminación o cambio de la relación en que se halla;

- La estrategia general, que se resume en ayudar a la mujer a "ser fiel a su propia experiencia de la relación para que pueda tomar decisiones coherentes con su realidad a cada paso del proceso"; y

- La táctica, que consiste en adaptar la estrategia dividiendo los objetivos en pasos intermedios en función del ritmo de la consultante y la regularidad con la que acude a atención.

Habiendo acumulado cierta experiencia aplicando esta propuesta, cerramos con las reflexiones de la última sesión de una terapia con una mujer que transitó el proceso de separación psicológica y ruptura de la relación violenta. Ante la pregunta de "¿qué es lo que más te sirvió de nuestras conversaciones y qué es lo que aprendiste?", la consultante respondió:

Creo que lo que me ayudó fue que me dabas una dosis de realidad. De hecho le decía a mi mamá cada vez que venía a sesión, "me voy a mi dosis de realidad". Me ayudaste a poner los pies en la tierra, a ir saliendo de las cosas que yo veía que no eran verdad, que eran ilusiones... Lo más importante que aprendí es a escucharme a mí y no dejarme de lado. Si siento, si pienso, si creo algo, tengo que decirlo, preguntarlo. Lo peor que puede pasar es que me equivoque y tenga que pedir disculpas. Pero no me voy a perder a mí misma de nuevo.

\section{Referencias}

Amor, P. J., Echeburúa, E., Corral, P., Zubizarreta, I. y Sarasua, B. (2002). Repercusiones psicopatológicas de la violencia doméstica en la mujer en función de las circunstancias del maltrato. Revista Internacional de Psicología Clínica y de la Salud, 2(2), 227-246.

Anderson, D. K. y Saunders, D. G. (2003). Leaving an abusive partner an empirical review of predictors, the process of leaving, and psychological well-being. Trauma, Violence, \& Abuse, 4(2), 163-191. https://doi. org/10.1177/1524838002250769

Blanco, P., Ruiz-Jarabo, C., García de Vinuesa, C. y Martín-García, M. (2004). La violencia de pareja y la salud de las mujeres. Gaceta Sanitaria, 18(4), 182-188.

Block, C. R. y DeKeseredy, W. S. (2007). Forced sex \& leaving intimate relationships: results of the Chicago women's health risk study. Women's Health and Urban Life, 6(1), 6-23.

Burkitt, K. y Larkin, G. (2009). The transtheoretical Model in Intimate Partner Violence Victimization: Stage Changes Over Time. En C. Murphy y R. Maiuro (Eds.) Motivational Interviewing and Stages of Change in Intimate Partner Violence (pp. 273-203). Springer. https://doi.org/10.1891/0886-6708.23.4.411

Burman, S. (2003). Battered women: Stages of change and other treatment models that instigate and sustain leaving. Brief Treatment and Crisis Intervention, 3(1), 83.

Campbell, J. C., Webster, D., Koziol-McLain, J., Block, C. R., Campbell, D. W., Curry, M. A., Gary, F., Glass, N., McFarlane, J., Sachs, C., Sharps, P., Ulrich, Y., Wilt, S. A. Manganello, J., Xu, X., Schollenberger, J., Frye, V. y Laughon, K. (2003). Risk factors for femicide in abusive relationships: Results from a 
multisite case control study. American Journal of Public Health, 93(7), 1089-1097. https://doi.org/10.2105/ ajph.93.7.1089

Cirici, R., Querol, N. y Ripoll, A. (2010). La consulta sanitaria: ¿un espacio privilegiado para la detección y el abordaje de la violencia de género? Formación médica continuada en atención primaria, 17(8), 550-559. https://doi.org/10.1016/s1134-2072(10)70213-9

Crowe, A. y Murray, C. E. (2015). Stigma from professional helpers toward survivors of intimate partner violence. Partner abuse, 6(2), 157-179. https://doi.org/10.1891/1946-6560.6.2.157

Cunillera, C. (2006). Personas con problemas de alcohol. Paidós.

Dalal, K. (2011). "Does Economic Empowerment Protect Women from Intimate Partner Violence?", Journal of Injury and Violence Research, 3(1), 35-44. https://doi.org/10.5249/jivr.v3i1.76

DeKeseredy, W. S., Donnermeyer, J. F., Schwartz, M. D., Tunnell, K. D. y Hall, M. (2007). Thinking critically about rural gender relations: Toward a rural masculinity crisis/male peer support model of separation/divorce sexual assault. Critical Criminology, 15(4), 295-311. https://doi.org/10.1007/ s10612-007-9038-0

DeKeseredy,W.S. y Joseph,C. (2006). Separation and/or divorce sexual assault in rural Ohio preliminary results of an exploratory study. Violence Against Women,12(3),301-311.https://doi.org/10.1177/1077801205277357

DeKeseredy, W. S., Rogness, M. y Schwartz, M. D. (2004). Separation/divorce sexual assault: The current state of social scientific knowledge. Aggression and Violent Behavior, 9(6), 675-691. https://doi.org/10.1016/j. avb.2003.08.004

Dobash, R. E., Dobash, R. P., Cavanagh, K. y Medina-Ariza, J. (2007). Lethal and nonlethal violence against an intimate female partner comparing male murderers to nonlethal abusers. Violence Against Women, 13(4), 329-353. https://doi.org/10.1177/1077801207299204

Enander, V. (2008). Women leaving violent men: Crossroads of emotion, cognition and action (Tesis doctoral). University of Gothenburg.

Enander, V. y Holmberg, C. (2008). Why does she leave? The leaving process (es) of battered women. Health Care for Women International, 29(3), 200-226. https://doi.org/10.1080/07399330801913802

Feder, G. S., Hutson, M., Ramsay, J. y Taket, A. R. (2006). Women exposed to intimate partner violence: expectations and experiences when they encounter health care professionals: a meta-analysis of qualitative studies. Archives of Internal Medicine, 166(1), 22-37. https://doi.org/10.1001/archinte.166.1.22

Ferrer, V. y Bosch, E. (2013). Del amor romántico a la violencia de género. Para una coeducación emocional en la agenda educativa. Profesorado, Revista de Currículum y Formación del Profesorado, 17(1), 105-122. https://doi.org/10.30827/profesorado.v23i2.9726

Griffing, S., Ragin, D. F., Sage, R. E., Madry, L., Bingham, L. E. y Primm, B. J. (2002). Domestic violence survivors' self-identified reasons for returning to abusive relationships. Journal of Interpersonal Violence, 17(3), 306-319. https://doi.org/10.1177/0886260502017003005

Henning, K. y Klesges, L. M. (2003). Prevalence and characteristics of psychological abuse reported by court-involved battered women. Journal of interpersonal violence, 18(8), 857-871. https://doi. org $/ 10.1177 / 0886260503253878$

Humphreys, J.,Lee, K., Neylan, T. y Marmar, C. (2001). Psychological and physical distress of sheltered battered women. Health care for women international, 22(4), 401-414. https://doi.org/10.1080/07399330152398927

Kelly, G. A. (1970). Behavior is an experiment. In D. Bannister (ed.), Perspectives in personal construct psychology. London: Academic.

Kelly, G. A. (2001). Psicología de los constructos personales: textos escogidos. Barcelona: Paidós.

Khaw, L. y Hardesty, J. L. (2007). Theorizing the process of leaving: turning points and trajectories in the stages of change. Family Relations, 56(4), 413-425. https://doi.org/10.1111/j.1741-3729.2007.00470.x

Khaw, L. y Hardesty, J. L. (2009). Leaving an abusive partner: Exploring boundary ambiguity using the stages of change model. Journal of Family Theory \& Review, 1(1), 38-53. https://doi.org/10.1111/j.17562589.2009.00004.x

Khaw, L. y Hardesty, J. L. (2014). Perceptions of boundary ambiguity in the process of leaving an abusive partner. Family process, 54(2), 327-343. https://doi.org/10.1111/famp.12104 
Kimmel, M. (2013). Angry White Men: American Masculinity at the End of an Era. Nation Books.

Krug, E. G., Dahlberg, L. L., Mercy, J. A., Zwi, A. B. y Lozano, R. (2002). Informe mundial sobre la violencia y la salud (p. 49). Organización Panamericana de la Salud (OPS) y Organización Mundial de la Salud (OMS). https://doi.org/10.1590/s1020-49892002001000002

Landenburger, K. M. (1989). A process of entrapment in and recovery from an abusive relationship. Issues in Mental Health Nursing, 10(3-4), 209-227. https://doi.org/10.3109/01612848909140846

Laso, E. (2017). Poder, agencia y comunión: obstáculos en la transformación de la masculinidad. En L. I. Castañeda y C. Alvizo (Coords.) Géneros, permanencias y transformaciones. Feminidades y masculinidades en el occidente de México (pp. 187-245). Universidad de Guadalajara.

Macias-Esparza, L. K. (2017). Dilemas implicativos y separación psicológica de mujeres en proceso de terminación de una relación violenta. Tesis doctoral. Universitat de Barcelona.

Macias-Esparza, L. K. y Laso, E. (2017) Una propuesta para abordar la doble ceguera: la Terapia Familiar Crítica sensible al Género. Revista de psicoterapia, 28(106), 129-148. https://doi.org/10.33898/rdp.v28i106.143

Mahoney, M. (2003). Constructive Psychotherapy: Theory and Practice. The Guildford Press.

McCloskey, L. A., Lichter, E., Williams, C., Gerber, M., Wittenberg, E. y Ganz, M. (2006). Assessing intimate partner violence in health care settings leads to women's receipt of interventions and improved health. Public health reports, 121(4), 435. https://doi.org/10.1177/003335490612100412

Midgett, A., Doumas, D., Sears, D., Lundquist, A. y Hausheer, R. (2015). A Bystander Bullying Psychoeducation Program with Middle School Students: A Preliminary Report. Professional Counselor, 5(4), 486-500. https://doi.org/10.15241/am.5.4.486

Miller, W. y Rollnick, S. (2013). Motivational Interviewing: Helping People Change (3a. Ed.). Guilford.

Morse, D. S., Lafleur, R., Fogarty, C. T., Mittal, M. y Cerulli, C. (2012). «They told me to leave»: How health care providers address intimate partner violence. The Journal of the American Board of Family Medicine, 25(3), 333-342. https://doi.org/10.3122/jabfm.2012.03.110193

Navarro, J. (2015). Violencia en las relaciones intimas. Una perspectiva clínica. Herder. https://doi.or$\mathrm{g} / 10.2307 / \mathrm{j} . \mathrm{ctvt} 9 \mathrm{k} 1 \mathrm{xf} .11$

Neimeyer, R. (2009). Constructivist Psychotherapy: Distinctive Features. Taylor and Francis.

Nicolaidis, C., Curry, M. y Gerrity, M. (2005). Health care workers' expectations and empathy toward patients in abusive relationships. The Journal of the American Board of Family Practice, 18(3), 159-165. https://doi. org/10.3122/jabfm.18.3.159

OECD (2014), Cerrando las brechas de género: Es hora de actuar. CIEDESS. https:// doi.or$\mathrm{g} / 10.1787 / 9789264208582$-es

ONU MUJERES (2013). Eliminación y prevención de todas las formas de violencia contra las mujeres y las niñas. Comisión de la Condición Jurídica y Social de la Mujer. $57^{\circ}$ periodo de sesiones. Nueva York.

Oram, S., Khalifeh, H. y Howard, L. M. (2016). Violence against women and mental health. The Lancet Psychiatry, 4, 159-170. https://doi.org/10.1016/s2215-0366(16)30261-9

Organización de las Naciones Unidas (ONU). (2008). Únete para poner fin a la violencia contra las mujeres. Departamento de Información Pública de las Naciones Unidas.

Organización Mundial de la Salud (1996). Resolución WHA49.25. Prevención de la violencia: una prioridad de la salud pública. Autor.

Organización Mundial de la Salud. (2001). Salud mental: nuevos conocimientos, nuevas esperanzas. Autor.

Organización Mundial de la Salud. (2005). Estudio multipaís sobre salud de la mujer y violencia doméstica. Autor.

Paz, J. I. (2011). Entender a la mujer que sufre violencia de género. En Nieto, C. La violencia de género en el contexto de la globalización. Artículo presentado en el Congreso «La violencia de género en el contexto de la globalización» (pp. 440-453). Universidad Internacional de Andalucía. http://publicaciones.unia.es/ busqueda-por-anno/item/la-violencia-de-genero-en-el-contexto-de-la-globalizacion

Peled, E., Eisikovits, Z., Enosh, G. y Winstok, Z. (2000). Choice and empowerment for battered women who stay: Toward a constructivist model. Social work, 45(1), 9-25. https://doi.org/10.1093/sw/45.1.9 
Pico-Alfonso, M. A., Garcia-Linares, M. I., Celda-Navarro, N., Blasco-Ros, C., Echeburúa, E. y Martínez, M. (2006). The impact of physical, psychological, and sexual intimate male partner violence on women's mental health: depressive symptoms, posttraumatic stress disorder, state anxiety, and suicide. Journal of Women's Health, 15(5), 599-611. https://doi.org/10.1089/jwh.2006.15.599

Prochaska, J. O. y DiClemente, C. C. (1982). Transtheoretical therapy: Toward a more integrative model of change. Psychotherapy: Theory, Research y Practice, 19(3), 276.

Prochaska, J. O. y DiClemente, C. C. (1994). The transtheoretical approach: Crossing traditional boundaries of therapy. Malabar Florida: Krieger Publishing.

Rees, K., Zweigenthal, V. y Joyner, K. (2014). Health sector responses to intimate partner violence: a literature review: original research. African Primary Health Care and Family Medicine, 6(1), 1-8. https://doi. org/10.4102/phcfm.v6i1.712

Rhatigan, D. L., Street, A. E. y Axsom, D. K. (2006). A critical review of theories to explain violent relationship termination: Implications for research and intervention. Clinical Psychology Review, 26(3), 321-345. https://doi.org/10.1016/j.cpr.2005.09.002

Robinson, L. y Spilsbury, K. (2008). Systematic review of the perceptions and experiences of accessing health services by adult victims of domestic violence. Health \& Social Care in the Community, 16(1), 16-30. https:// doi.org/10.1111/j.1365-2524.2007.00721.x

Roca-Cortés, N, Espín, J., Rosich, M., Cantera, L. y Strey, M. (2005). Cambios de creencias e intervención en mujeres que sufren maltrato. Revista Electrónica de la Universitat Jaume I, 1(1), 143-155.

Roca-Cortés, N., Paíno, M., Albarracín, M., Cordoba, L. y Espín, J. (2007). Treball grupal i violència sexista. Investigació-acció d'un grup de suport psicosocial a dones. Ajuntament de Barcelona, Serveis Personals.

Roca-Cortés, Porrúa, C. yepes, M., Martínez, M., Codina, M., Fernández, A., Ruiz, S. y Cremades, M. (2015). Recuperación de mujeres en situación de violencia machista. Descripción e instrumentación. Ayuntamiento de Barcelona, Universitat de Barcelona y Fundación Salud y Comunidad.

Rodríguez Gimena, M., Pérez Alonso, E., Moratilla Monzó, L., Escortell Mayor, E., Lasheras Lozano, L. y Domínguez Bidagor, J. (2008). Guía de apoyo en atención primaria para abordar la violencia de pareja hacia las mujeres. Dirección General de Salud Pública y Alimentación. Consejería de Sanidad.

Sagot, M. y Carcedo, A. (2000). La ruta crítica de las mujeres afectadas por la violencia intrafamiliar en América Latina. Organización Panamericana de la Salud, Programa Mujer, Salud y Desarrollo.

Scheffer, M. y Renck, B. (2008). Intimate partner violence and the leaving process: Interviews with abused women. International Journal of Qualitative Studies on Health and Well-being, 3(2), 113-124. https://doi. org/10.1080/17482620801945805

Slive, A. y Bobele, M. (2013). Cuando sólo tienes una hora: terapia efectiva para clientes de atención inmediata. Paidós.

Trevillion, K., Corker, E., Capron, L. E. y Oram, S. (2016). Improving mental health service responses to domestic violence and abuse. International review of psychiatry, 28(5), 423-432. https://doi.org/10.1080/0954 0261.2016 .1201053

UNODC (Oficina de las Naciones Unidas contra la Droga y el Delito). (2013). Estudio Mundial sobre el homicidio. Vienna: United Nations Publication.

Vyas, S. y Watts, C. (2009). How does economic empowerment affect women's risk of intimate partner violence in low and middle income countries? A systematic review of published evidence. Journal of International Development: The Journal of the Development Studies Association, 21(5), 577-602.

Velzeboer, M., Ellsberg, M., Clavel-Arcas, C. y García-Moreno, C. (2003). Violencia contra las mujeres: responde el sector salud. Washington, D.C.: Organización Panamericana de la Salud.

Walker, R., Logan, T., Jordan, C. E. y Campbell, J. C. (2004). An integrative review of separation in the context of victimization consequences and implications for women. Trauma, Violence, \& Abuse, 5(2), 143-193. https://doi.org/10.1177/1524838003262333

World Economic Forum. (2020). The global gender gap report. Geneva: World Economic Forum. http://www3. weforum.org/docs/WEF GGGR 2020.pdf 
World Health Organization. (2013). Global and regional estimates of violence against women: prevalence and health effects of intimate partner violence and non-partner sexual violence. Autor.

Wuest, J. y Merritt-Gray, M. (1999). Not going back sustaining the separation in the process of leaving abusive relationships. Violence Against Women, 5(2), 110-133. https://doi.org/10.1177/1077801299005002002

Artículo recibido: 24/04/2019

Artículo aceptado: 07/02/2020 